\title{
3 Research Square \\ The Mechanism of Aidi Injection on Breast Cancer Based on Network Pharmacologyanalysis
}

Jing Su

Shaanxi University of Chinese Medicine

Kedi Liu

Shaanxi University of Chinese Medicine

Xingru Tao

Shaanxi University of Chinese Medicine

Fei Li

Fourth Military Medical University

Shi Zhao

Shaanxi University of Chinese Medicine

Yang Bai

National Drug Clinical Trial Institute,the Second Affiliated Hostipal,Shananxi University of Chinese Medicine.

Xinming Lu

Youyi Clinical Laboratories of Shaanxi

Jing Li

Youyi Clinical Laboratories of Shaanxi

Sha Chen

Youyi Clinical Laboratories of Shaanxi

Jialin Duan

Department of Pharmacy,Xijing Hospital.

\section{Peifeng Wei}

National Drug Clinical Trial Institute冈the Second Affiliated Hostipal,Shaanxi University of Chinese Medicine.

Miaomiao xi ( $\nabla$ miaomiaoxi2014@163.com )

TANK Medicinal Biology Institute of Xi'an

\section{Research}

Keywords: Breast Cancer, Aidi injection, Network pharmacology

Posted Date: October 8th, 2020 
DOI: https://doi.org/10.21203/rs.3.rs-85981/v1

License: (c) (1) This work is licensed under a Creative Commons Attribution 4.0 International License. Read Full License 


\section{Abstract}

Background: Aidi injection (ADI)is a Chinese patent medicine with anti-cancer effect, which has been used to treat breast cancer (BC) in China for many years, but its potential pharmacological mechanism is still indeterminacy. In this resaearch, network pharmacology, a systematic and comprehensive approach, was used to reveal ADI's potential pharmacological mechanism in treating $B C$ for the first time.

Methods: Databases were used to collect targets related to the bioactive components of ADI and BC. the relevant networks were established by the string database, and were screened potential bioactive components and core targets. Eventually, core targets and pathway enrichment were analyzed by DAVID database.

Results: As the results showed, we collected 99 bioactive ingredients,345 ADI-related targets after deduplication and $368 \mathrm{BC}$-related targets. Of these, 108 common targets were overlapped. We then performed an enrichment analysis on the common target network and the protein-protein interaction (PPI) network.

Conclusion: The results showed that ADI may inhibit breast cancer through seven important signal pathways involved in the "regulation of vascular endothelial function", "inflammatory response" and "apoptosis" biological processes. Through further clustering and enrichment analysis of the PPI network of ADI's bioactive component targets and BC-related targets, we found that cancer, ErbB, MAPK, TLR, chemokine, p53 and cell cycle signaling pathway, mainly contributed to the effects of ADI in treating BC. In conclusion, this study reveals the possible mechanism of $A D I$ in treating $B C$, and provides a new direction for drug development for $A D I$ in treating $B C$.

\section{Background}

The incidence and mortality of cancer have increased year by year, and cancer has become the leading cause of death ${ }^{[1]}$. Among women, $\mathrm{BC}$ have the highest incidence in recent years and are on the rise, which seriously threatening their health ${ }^{[2]}$.Worldwide, there are about 2.1 million newly diagnosed female $\mathrm{BC}$ cases in 2018, accounting for almost 1 in 4 cancer cases among women ${ }^{[3]}$. The disease is the most frequently diagnosed cancer in the vast majority of the countries (154 of 185) and is also the leading cause of cancer death in over 100 countries $^{[4]}$.In clinic, radiation therapy, chemotherapy, endocrine therapy, photodynamic therapy囚and targeted therapy are commonly used. However, they have some disadvantages ${ }^{[5]}$. For example, radiotherapy will induce large gastrointestinal reactions, obvious decline in blood imageradiation dermatitis, radiation pneumonia, and so on ${ }^{[6]}$. Another example is that in neoadjuvant chemotherapy, patients who are not sensitive to chemotherapy, which will expand the scope of surgery, increase the difficulty of surgery, and may even lose the opportunity of surgery.

Chinese Herbal Medicine has been one of the most frequently used alternative treatments for different types of cancer. Chinese medicine has been paid more and more attention by doctors and patients. For 
patients who have received systemic adjuvant chemotherapy, traditional Chinese medicine treatment can effectively combine with western medicine to alleviate toxic side effects caused by chemotherapy drugs, such as blood cell reduction, nausea, vomiting, diarrhea, rash, hair loss, fatigue, etc ${ }^{[7-8]}$. Many laboratory studies have shown that crude extracts of Chinese herbal medicines and active ingredients can inhibit BC cell proliferation and migration, induce apoptosis of breast cancer cells in vitro, and reduce the side effects of chemotherapy drugs ${ }^{[9-11]}$.

Aidi injection (ADI, Z52020236, China food and Drug Administration) includes Mylabrisphalerata Pallas (Ban Mao), Acanthopanax senticosus(Ci Wu Jia),Astragalus membranaceus(Huang Qi) and Panax ginseng (Ren Shen) $\llbracket$ which has the effects of clearing away heat, detoxifying, eliminating phlegm and dispersing stasis. Their main active ingredients are cantharidin, astragaloside, ginsenoside, elentheroside $E$, isofraxidin, syringin, coniferin and so on. ADI has been used to treat the cancers, including gynecological malignancies, primary liver cancer, lung cancer, $B C$ etc ${ }^{[12-14]}$.Studies have shown that ADI can inhibit proliferation, promote apoptosis and necrosis of BC cells, and significantly reduce cell diameter ${ }^{[15]}$.In addition, it can reduce the serum VEGF level in BC patients, and inhibit tumor angiogenesis, thereby inhibiting tumor cell proliferation, invasion and metastasis ${ }^{[16]}$. However, its mechanism is largely unknown to us.

It can also be seen that Chinese medicine has the characteristics of multiple components and multiple targets, and in previous studies, comprehensive data could not be obtained. In recent years, network pharmacology has integrated the multidisciplinary technologies and contents of systems biology, multidirectional pharmacology, bioinformatics and computer science to carry out "disease phenotype-genetarget-drug". By establishing a multi-level network, we explored the relationship between drugs and diseases, clarified the mechanism of drug action, and studied its integrity, systemi city, and complexity. The results may guide the development of new drugs. This research method is consistent with the characteristics of traditional Chinese medicine ${ }^{[13]}$. To construct the "drug component-target" multi-level biological network of ADI, network pharmacology method was used in this study.

\section{Methods}

\subsection{Collecting drug molecular information and screening for active molecules}

Chemical compounds in ADI were mainly obtained from the TCM systems pharmacology database (TCMSP, http://tcmspw.com/tcmsp.php) and the TCM database@Taiwan (TCM@Taiwan, http://tcm.cmu.edu.tw), the two largest pharmacological data platforms for Traditional Chinese Medicine. They contain all herbs, chemical components and pharmacokinetic properties (namely, absorption, distribution, metabolism and excretion or ADME) information in the Pharmacopoeia of the People's Republic of China (2010 edition) ${ }^{[17]}$. In addition, the databases of the China National Knowledge Infrastructure were also used to supply any omitted components. Finally,313 components from ADI were 
collected, including 190, 87, 9 and 27 from Renshen, Huangqi, Ciwujia, and Banmao, respectively (Supplementary Table S1)

\subsection{Pharmacokinetics ADME Assessment}

The recommended standard refers to the TCMSP database (TCMSP, http://ibts.hkbu.edu.hk/LSP/tcmsp.php), which includes only predicting the octanol-water partition coefficient (AlogP) and drug similarity (DL). If $A \log P<5$ and $D L \geq 0.18$ is satisfied, when the compound is retained, the compound that didn't not conform to the standard compound would be removed from the list. The final compound was obtained. (Supplementary Table S2).

The screened compound molecules were then searched in the Pubchem database (https://pubchem.ncbi.nlm.nih.gov/) and the found compounds in the PubchemCID number and SMILES format were recorded with molecular SDF file is saved. The molecules which were not found in Pubchem, in the Swiss Target Prediction database (http://www.swisstargetprediction.ch/), drew the same structure according to the formula in the TCMSP database, then recorded the SMILES format and saved the SDF file of the molecule.

\subsection{Prediction of compound-related target genes}

We upload the SDF file saved in the previous step to the pharmmapper database (http://www.lilabecust.cn/pharmmapper/), and fill in the mailbox, then continue to select "Human protein targets only", and finally get all predictions molecularly related targets. We then found the official name of the predicted gene on the UniProt website (http://www.uniprot.org/) and selected "Search / ID Mapping", selecting the type as "Homo sapiens". Finally, our various ID forms will be converted to UniProt ID. This data is arranged into the ADI's drug component-target relationship data set (SupplementaryTable3).

\subsection{Obtaining genetic data related to $\mathrm{BC}$}

BC-related genes are available from two official databases: Gene Cards database (https://www.genecards.org/), online Mendelian inheritance (OMIM, http ://www.omim.org/) and the simple "Homo sapiens" chose the protein linked to BC (SupplementaryTable4).

\subsection{Network construction}

We followed the method of $\mathrm{Li}$ et al to continue the following work according to the previous work in our laboratory ${ }^{[18]}$, and we used Cytoscape (http://www.cytoscape.org, version 3.7.1) ${ }^{[19]}$ to construct the common-target network selected from ADI and BC (Supplementary Table5). 
PPI network construction. Protein is a biological macromolecule. Organisms have the synergistic effect of protein to complete various life activities and achieve various life functions. The various functions of an organism are manifested by the interaction of many proteins under specific conditions rather than by individual proteins. Establishment of PPI networks can make the link between prediction targets and other human proteins closer, which has become a promising target for drug discovery ${ }^{[20]}$. Using Cytoscape plug-in Bisogenet to build and visually analyze at different detail levels, the network contains six main PPI databases, including the biomolecular interaction network database (BIND), the complete molecular interaction database (complete), the human protein reference database (HPRD), the molecular interaction database (MINT), and the biological interaction database master database (Biogrid) ${ }^{[21]}$. We define the identifier as "Homo sapiens, protein identifier only" in the Bisogenet program, select the PPI data sources, set the distance from the input set to the new nodes as "1", and represent the output as "proteins". We establish PPI networks with BC related goals and ADI related goals.

Central network evaluation. Along with the rapid development of bioinformatics technology, the evaluation of the central network of PPI networks containing a large number of gene combinations and proteomics data becomes the main method to screen the core proteins. The PPI network BC related goals and ADI related goals is merged and then intersected. Then we use the plug-in of the CytoNCA evaluate intersection. Cytonca includes six centriality measures, such as degree centrality (DC), closed centrality $(\mathrm{CC})$, network centrality (NC), Betweenness centrality (BC), method based on local average connectivity (LAC) and eigenvector centrality (EC) ${ }^{[22]}$, to filter the data. We take the "DC $\geq 2 \times$ median DC" as the screening criteria for preliminary processing of data, and the criterion used for secondary screening is "DC $\square \mathrm{BC} \square \mathrm{EC} \square \mathrm{CC} \square \mathrm{LAC} \square \mathrm{NC}$ greater than or equal to their median"[23] (Supplementary Table 6) for data screening as the core target.

Cluster analysis. Clustering analysis can be performed by extracting nodes with the same or similar attributes as clusters for sub-regional analysis of complex PPI networks. The core-target PPI network (Supplementary Table 7) conducted cluster analysis by MCODE, a cluster analysis algorithm in Cytoscape in this research ${ }^{[24,25]}$.

\subsection{Enrichment analysis}

Annotated Visualization Database and Integrated Discovery Database (DAVID, https://david.nicifcrf.gov/, version 6.8) with $P \leq 0.05$ as screening criteria were used for gene ontology (GO) enrichment analysis ${ }^{[26,}$ 27]. We used the DAVID database to apply for Kyoto Encyclopedia of Genes and Genomes (KEGG) pathway enrichment analysis with $\mathrm{p} \leq 0.05$ as screening criteria ${ }^{[28]}$ to reveal the gene pathway annotation network for functional groupings. Furthermore, a collection of tools for "Search Pathway" and "Color Pathway ", KEGG Mapper (https://www.genome.jp/kegg/mapper.html) which is used to analyze the connections between upstream and downstream in main signaling pathways ${ }^{[29]}$. The mechanism of ADI therapeutic BC (Supplementary Table S8, 9, 10) is revealed by enrichment analysis of common target networks and PPI networks. 


\section{Results}

\subsection{ADI component-target network}

We collected 313 ADI components from the TCMSP and TCM@Taiwan natural products database, of which 190 were from Renshen, 87 from Huangqi and 9 from Ciwujia,27 from Banmao. The components were screened with the criteria of AlogP $<5$ and $D L \geq 0.18$, and 99 bioactive components of $A D I$ were included, of which 47.5\% (47/99) were from renshen,40.4\% (40/99) from Huangqi,9.1\% (9/99) from Ciwujia and 3.0\% (3/99) from Banmao. (Fig.1A,B). Of these 99 bioactive components, three groups from Renshen, Huangqi, and Ciwujia overlapped significantly, indicating that different herbs in a formula could share the same or similar components and targets with synergistic effects. Generally speaking, the therapeutic and preventive effects of a compound formula rely on the synergies among the multiple components, targets and pathways ${ }^{[30]}$.Subsequently, the structure information of bioactive components such as molecular structures, canonical smiles and their "sdf" files is obtained from Pubchem and ZINC product databases. After removing duplicate samples, 351 potential targets were explored from 99 bioactive ingredients. Then, we used Cytoscape to build a visual ADI target network with 443 nodes and 863 edges (Fig. 1C).

\subsection{Common-target network}

We input the 108 common targets into the DAVID for GO and KEGG analysis to obtain 71 biological processes (BP), 12 cellular components (CC), 22 molecular functions (MF), and 81 KEGG pathways $(P<0.05)$ to understand the mechanism of $A D I$ treatment $B C(F i g .2 A, B)$.

We found that the enrichment pathways were cancer pathways, ErbB signaling pathways, and MAPK signaling, which are closely related to the occurrence of BC. In addition, we found that ADI inhibited tumorigenesis by regulating the biological processes of nitric oxide, controlling cell proliferation and apoptosis, and inhibiting angiogenesis. In addition, the positive regulation of the ERK1 and ERK2 cascades is also considered to be closely related to cell proliferation, transformation, and differentiation, etc., and is of great significance to elucidate the pathogenesis of BC.

\subsection{ADI-BC PPI network}

Bisogenet established a PPI network ADI related objectives and BC related objectives (Fig. 3A, B). Then the same nodes and edges in two PPI networks will obtain an intersection point (Fig. 3C). Next, the intersection of the PPI network is evaluated CytoN selecting topology analysis. The significant target of ADI on BCPPI network is selected by the "DC>=44"'" screening criterion (Fig. 3D). Six screening criteria were selected for further screening, and a core target PPI network containing 423 ADI candidate targets was obtained. 


\subsection{Enrichment analysis of the $\mathbf{4 2 2}$ key targets.}

We elucidated the biological functions of 422 major targets by dividing the final central PPI network into eight clusters and selecting GO and KEGG pathways for enrichment analysis, respectively. A total of 379 $\mathrm{BPs}, 67 \mathrm{CCs}$, and $92 \mathrm{MFs}$ were obtained. Based on the results of these $\mathrm{GO}$ terms, it could be seen that the development of $\mathrm{BC}$ was closely related to various biological processes, such as angiogenesis, cell proliferation, apoptosis, inflammatory response, ubiquitinated proteins, etc. The KEGG enrichment results of these eight clusters overlapped with the KEGG enrichment results of 108 common targets.It was found that the ADI enrichment pathway mainly focused on the cancer, ErbB, MAPK, TLR, chemokine, p53 and cell cycle signaling pathway.(Fig.4)

\section{Discussion}

$\mathrm{BC}$ is one of the most common diseases in the female population [31]. It poses a serious threat to human health, especially women's health. Facing this complex disease, traditional Chinese medicine (TCM) with multiple components and multiple targets is an important and commonly used adjuvant therapy in China. TCM can not only relieve the discomfort of cancer, but also can be used throughout the treatment process. Studies have shown encouraging results with traditional Chinese medicine for breast cancer ${ }^{[32,33]}$. The ADI we studied was composed by Renshen,Huangqi,Ciwujia and Banmao. In 2000, it was identified as a secondary protection Chinese medicine and a National Medical Insurance Class B drug $^{[34]}$. In clinical, ADI has been used to treat various malignant tumors, including BC. However, its biologically active ingredients and mechanism are still unclear, which greatly limits its clinical application. With the rapid development of bioinformatics, network pharmacology has become an effective method to study the relationship between biologically active ingredients and the mechanism of TCM.

In this study, we focused on three aspects of network pharmacology (Fig.5). First $\$ the occurrence of cancer and some other diseases were found in the enrichment analysis of 108 common targets. Second, exhibited a refined detail of ADI on BC compared to the common-target analysis. Third, at the gene level, cancer pathway, ErbB, MAPK, TLR, chemokine, p53 and cell cycle signaling pathway are considered to be the key pathways for treating $B C$.

We then searched for seven key signaling pathways of $A D I$ related to $B C$ treatment. Cancer pathways can be found to be directly related to cancer. Many studies have found that breast cancer may be caused by regulating the ErBb signaling pathway ${ }^{[35]}$. By reducing the phosphorylation of the receptor tyrosine kinases ErbB1, ErbB2, and ErbB3, the ErbB signaling pathway can be inhibited, thereby suppressing the occurrence of breast cancer ${ }^{[36]}$. The core genes involved in this pathway are EGRF and Akt1. EGRF is one of the ErBbs receptor tyrosine kinases. Studies have shown that tumor growth and metastasis can be inhibited by inhibiting EGF-induced EGFR signaling pathway and its downstream targets ${ }^{[37]}$. Akt plays important biological roles, such as participating in cell survival, apoptosis, proliferation, angiogenesis and 
other physiological processes and tumorigenesis ${ }^{[38]}$.Studies have found that down-regulating Akt phosphorylation inhibits tumor development. After ErbB2 and ErbB2 receptors bind in the ErBb signaling pathway, they activate intracellular phosphatase activity, then phosphorylate PIP2 to generate PIP3, and recruit Akt proteins to the inner surface of the cell membrane. In our research, we also found that chemokines are closely related to tumorigenesis and growth. Through targeted MAPK signaling pathways and chemokine signaling pathways, It could be seen that they were abnormally expressed in many malignancies and played a vital role in the process of metastasis. Chemokines regulate tumor growth by affecting tumor stromal cells, inducing cell growth in the tumor microenvironment, and releasing angiogenic factors ${ }^{[39,40]}$.The AKT signaling pathway is an intracellular mechanism of chemokinemediated VEGF-C secretion in human breast cancer cells ${ }^{[41]}$. In promoting tumorigenesis and development, chronic infection and inflammation are considered the most important acquisition and environmental factors. The Toll-like receptor signal transduction pathway is related to inflammation. It can be considered that inflammation is also one of the mechanisms leading to breast cancer. The P53 signaling pathway is the major pathway in cell cycle control, and account for about $50 \%$ of human malignancies ${ }^{[42]}$.

The polymorphism of human genetic genes determines the human body's sensitivity and response to diseases and drugs, and the diversity of clinical manifestations. This is similar to the concept of "Zheng" (pattern) in TCM-a summary of pathological characteristics at a certain stage of disease development, and it is a functional state in which the body responds to pathogenic factors. Based on this, we speculate that one of the theoretical foundations of TCM is natural polygenes ${ }^{[43,44]}$. We believe that ADI is effective for breast cancer from the level of gene repair and expression to the level of signal transduction and cascade activation in the pathway. Meanwhile, the relationship between human genetic polymorphism and TCM model type/treatment effect was emphasized by some researchers from the perspective of clinical and experimental trials ${ }^{[45]}$. Therefore, a new research method to verify the effectiveness of TCM may be polygenic analysis. However, our study still has some limitations. First, our collection of bioactive components and targets is not comprehensive enough. we modified it by new detection methods such as liquid chromatography, mass spectrometry, two-dimensional liquid chromatography or quadrupole timeof-flight mass spectrometry. Second, our online pharmacology lacks validation of animal and clinical trials. In the future, the relationship between TCM model type and genetic polymorphism will be verified in animal experiments and clinical trials.

\section{Conclusions}

According to the results, the therapeutic effects of ADI were mainly through seven important signaling pathways in three biological processes, namely"regulating vascular endothelial function","inflammatory response" and "apoptosis". Among them, ErbB signaling pathway, chemokine signaling pathway, p53 signaling pathway, and cell cycle can be considered as the main pathways for ADI to treat breast cancer. This study reveals the potential mechanism of action of $A D I$ on in treating $B C$ to some extent. It also provides a new direction for drug development for ADI in treating BC. 


\section{Abbreviations}

ADI: Aidi injection, BC: Breast Cancer, TCM: traditional Chinese medicine, TCMSP: Traditional Chinese Medicine Systems Pharmacology, AlogP: the octanol-water partition coefficient, DL: drug-likeness, BPs: biological processes, CCs: cell compositions, MFs: molecular functions, ADME: absorption, distribution, metabolism and excretion, OMIM: online Mendelian inheritance, PPI: protein protein interaction, BIND: biomolecular interaction network database, HPRD: the human protein reference database, MINT: the molecular interaction database, DC: degree centrality, CC: closed centrality, NC: network centrality, BC: Betweenness centrality, LAC: local average connectivity, EC: eigenvector centrality, GO: gene ontology, KEGG: Kyoto Encyclopedia of Genes and Genomes,PI3K: phospholipid phthalinositol kinase, MAPK: mitogen-activated protein kinase, AKT: protein kinase B, MMP2: matrix metalloproteinase 2, MMP9: matrix metalloproteinase 9,TLR: Toll like receptors.

\section{Declarations}

\subsection{Ethics approval and consent to participate}

Not applicable.

\subsection{Consent for publication}

Not applicable.

\subsection{Availability of data and materials}

The data used to support the results of this study can be obtained from the first author upon reasonable request.

\subsection{Competing interests}

The authors declare that they have no conflict of interest.

\subsection{Funding}

This study is supported by the Subject Innovation Team of the Second Affiliated Hospital of Shaanxi University of Chinese Medicine (NO. 2020XKTD-A04区.

\subsection{Authors' contributions}


Jing Su: Investigation, Methodology, Writing - original draft; Kedi Liu: Investigation, Methodology, Writingoriginal draft; Xingru Tao: Investigation, Methodology, Writing - original draft; Fei Li: Resources, Software; Shi Zhao: Resources, Software; Yang Bai: Resources, Software; Xinming Lu: Data curation; Jing Li: Data curation; Sha Chen: Data curation; Jialin Duan: Writing - review \& editing; Peifeng Wei: Writing - review \& editing; Miaomiao Xi: Funding acquisition, Project administration, Writing - review \& editing.

\subsection{Acknowledgements}

Not applicable.

\section{References}

1. Chen W, Zheng R, Baade PD, et al. Cancer statistics in China, 2015. CA Cancer J Clin. 2016;66(2):115132. doi: $10.3322 /$ caac. 21338

2. Allemani C, Weir HK, Carreira H, et al. Global surveillance of cancer survival 1995-2009: analysis of individual data for 25,676,887 patients from 279 population-based registries in 67 countries (CONCORD-2) [published correction appears in Lancet. 2015 Mar 14;385(9972):946]. Lancet. 2015;385(9972):977-1010. doi:10.1016/S0140-6736(14)62038-9

3. Schneider AP 2nd, Zainer CM, Kubat CK, et al. The breast cancer epidemic: 10 facts. Linacre Q. 2014;81(3):244-277. doi:10.1179/2050854914Y.0000000027

4. Bray F, Ferlay J, Soerjomataram I, et al. Global cancer statistics 2018: GLOBOCAN estimates of incidence and mortality worldwide for 36 cancers in 185 countries [published correction appears in CA Cancer J Clin. 2020 Jul;70(4):313]. CA Cancer J Clin. 2018;68(6):394-424. doi:10.3322/caac. 21492

5. Mao Y, Prediction of network pharmacological mechanism of Chinese medicine for metastatic breast cancer,Tianjin Medical University.

6. Qi F, Zhao L, Zhou A, et al. The advantages of using traditional Chinese medicine as an adjunctive therapy in the whole course of cancer treatment instead of only terminal stage of cancer. Biosci Trends. 2015;9(1):16-34. doi:10.5582/bst.2015.01019

7. Li X, Yang G, Li X, et al. Traditional Chinese medicine in cancer care: a review of controlled clinical studies published in chinese [published correction appears in PLoS One.2013;8(6).doi:10.1371/annotation/b53a0b8b-3eb6-44a2-9c37-bc9bb66bfe7e]. PLoS One. 2013;8(4):e60338. doi:10.1371/journal.pone.0060338

8. Yang X, Hao J, Zhu CH, et al. Survival Benefits of Western and Traditional Chinese Medicine Treatment for Patients With Pancreatic Cancer. Medicine (Baltimore). 2015;94(26):e1008. doi:10.1097/MD.0000000000001008

9. Hsu RJ, Hsu YC, Chen SP, et al. The triterpenoids of Hibiscus syriacus induce apoptosis and inhibit cell migration in breast cancer cells. BMC Complement Altern Med. 2015;15:65. Published 2015 Mar 
10. Tan X, Guo L, Wang G. Polyporus umbellatus inhibited tumor cell proliferation and promoted tumor cell apoptosis by down-regulating AKT in breast cancer. Biomed Pharmacother. 2016;83:5 26-535. doi:10.1016/j.biopha.2016.06.049

11. Wu C, Lai JN, Tsai YT. The prescription pattern of Chinese herbal products that contain dang-qui and risk of endometrial cancer among tamoxifen-treated female breast cancer survivors in Taiwan: a population-based study. PLoS One. 2014;9(12):e113887. Published 2014 Dec 8. doi:10.1371/journal.pone.0113887

12. Zeng YB, Liu XL, Li CJ, et al.Chemical constituents from Mylabris phalerata and their cytotoxic activity in vitro. Chin J Chin Mater Med. 2016;1(모):859-863. doi:10.4268/cjcmm20160516

13. Wang D, Chen Y, Ren J, et al. A randomized clinical study on efficacy of Aidi injection combined with chemotherapy in the treatment of advanced non-small cell lung cancer.Chin $\mathrm{J}$ Lung Cancer. 2004;7(3):247-249.

14. Sun, J. B. , Xu, P. R. , Shi, D. L. , et al. Evaluation on clinical efficacy of aidi injection combined with gp chemotherapy in treatment of advanced non-small cell lung cancer in elder patients. J Jilin Univ Med Edit, 38(1), 151-154.

15. Tang J, Wang G. Antitumor Effect of Aidi Injection on ErbB2-positive Breast Cancer cells and mechanisms of Action." Pharm J Chin PLA. v.34(04):49-52+56.

16. Wang M, Wu L. "Effect of Adi injection on serum VEGF in patients with breast cancer". Chin J Clin Res. 2013(11):18-19+25.

17. Ru J, Li P, Wang J, et al. TCMSP: a database of systems pharmacology for drug discovery from herbal medicines. J Cheminform. 2014;6:13. Published 2014 Apr 16. doi:10.1186/1758-2946-6-13

18. Li F, Duan J, Zhao M, et al. A network pharmacology approach to reveal the protective mechanism of Salvia miltiorrhiza-Dalbergia odorifera coupled-herbs on coronary heart disease. Sci Rep. 2019;9(1):19343. Published 2019 Dec 18. doi:10.1038/s41598-019-56050-5

19. Shannon P, Markiel A, Ozier O, et al. Cytoscape: a software environment for integrated models of biomolecular interaction networks. Genome Res. 2003;13(11):2498-2504. doi:10.1101/gr.1239303

20. Murakami Y, Tripathi LP, Prathipati P, et al. Network analysis and in silico prediction of protein-protein interactions with applications in drug discovery. Curr Opin Struct Biol. 2017;44:134-142. doi:10.1016/j.sbi.2017.02.005

21. Martin A, Ochagavia ME, Rabasa LC, et al. BisoGenet: a new tool for gene network building, visualization and analysis. BMC Bioinformatics. 2010;11:91. Published 2010 Feb 17. doi:10.1186/1471-2105-11-91

22. Tang Y, Li M, Wang J, et al. CytoNCA: a cytoscape plugin for centrality analysis and evaluation of protein interaction networks. Biosystems. 2015;127:67-72. doi:10.1016/j.biosystems.2014.11.005

23. Song W, Ni S, Fu Y, et al. Uncovering the mechanism of Maxing Ganshi Decoction on asthma from a systematic perspective: A network pharmacology study. Sci Rep. 2018;8(1):17362. Published 2018 Nov 26. doi:10.1038/s41598-018-35791-9 
24. Ahmed HA, Bhattacharyya DK, Kalita JK. Core and peripheral connectivity based cluster analysis over PPI network. Comput Biol Chem. 2015;59 Pt B:32-41. doi:10.1016/j.compbiolchem.2015.08.008

25. Bader GD, Hogue CW. An automated method for finding molecular complexes in large protein interaction networks. BMC Bioinformatics. 2003;4:2. doi:10.1186/1471-2105-4-2

26. Huang da W, Sherman BT, Lempicki RA. Systematic and integrative analysis of large gene lists using DAVID bioinformatics resources. Nat Protoc. 2009;4(1):44-57. doi:10.1038/nprot.2008.211

27. Huang da W, Sherman BT, Lempicki RA. Bioinformatics enrichment tools: paths toward the comprehensive functional analysis of large gene lists. Nucleic Acids Res. 2009;37(1):1-13. doi:10.1093/nar/gkn923

28. Bindea G, Mlecnik B, Hackl H, et al. ClueGO: a Cytoscape plug-in to decipher functionally grouped gene ontology and pathway annotation networks. Bioinformatics. 2009;25(8):1091-1093. doi:10.1093/bioinformatics/btp101

29. Kanehisa M, Furumichi M, Tanabe M, et al. KEGG: new perspectives on genomes, pathways, diseases and drugs. Nucleic Acids Res. 2017;45(D1):D353-D361. doi:10.1093/nar/gkw1092

30. Li S, Zhang B. Traditional Chinese medicine network pharmacology: theory, methodology and application. Chin J Nat Med. 2013;11(2):110-120. doi:10.1016/S1875-5364(13)60037-0

31. Ling CQ, Wang LN, Wang $Y$, et al. The roles of traditional Chinese medicine in gene therapy. J Integr Med. 2014;12(2):67-75. doi:10.1016/S2095-4964(14)60019-4

32. Qi F, Zhao L, Zhou A, et al. The advantages of using traditional Chinese medicine as an adjunctive therapy in the whole course of cancer treatment instead of only terminal stage of cancer. Biosci Trends. 2015;9(1):16-34. doi:10.5582/bst.2015.01019

33. Li Y, Zhu X, Bensussan A, et al. Herbal Medicine for Hot Flushes Induced by Endocrine Therapy in Women with Breast Cancer: A Systematic Review and Meta-Analysis. Evid Based Complement Alternat Med. 2016;2016:1327251. doi:10.1155/2016/1327251

34. Zhao Y,"Meta Analysis of Traditional Chinese Medicine Injection Combined with Chemotherapy for Advanced Breast Cancer".Beijing University of Chinese Medicine .2019.

35. Ghaemi Z, Soltani BM, Mowla SJ. MicroRNA-326 Functions as a Tumor Suppressor in Breast Cancer by Targeting ErbB/PI3K Signaling Pathway. Front Oncol. 2019;9:653. Published 2019 Jul 30. doi:10.3389/fonc. 2019.00653

36. Hardy KM, Booth BW, Hendrix MJ, et al. ErbB/EGF signaling and EMT in mammary development and breast cancer. J Mammary Gland Biol Neoplasia. 2010;15(2):191-199. doi:10.1007/s10911-0109172-2

37. Gennari A, Conte P, Rosso R, et al. Survival of metastatic breast carcinoma patients over a 20-year period: a retrospective analysis based on individual patient data from six consecutive studies. Cancer. 2005;104(8):1742-1750. doi:10.1002/cncr.21359

38. Lee YW, Chen TL, Shih YR, et al. Adjunctive traditional Chinese medicine therapy improves survival in patients with advanced breast cancer: a population-based study. Cancer. 2014;120(9):1338-1344. doi:10.1002/cncr.28579 
39. Tutunea-Fatan E, Majumder M, Xin X, et al. The role of CCL21/CCR7 chemokine axis in breast cancerinduced lymphangiogenesis. Mol Cancer. 2015;14:35. Published 2015 Feb 10. doi:10.1186/s12943015-0306-4

40. Bancovik J, Moreira DF, Carrasco D, et al. Dermcidin exerts its oncogenic effects in breast cancer via modulation of ERBB signaling. BMC Cancer. 2015;15:70. Published 2015 Feb 19. doi:10.1186/s12885-015-1022-6

41. Zhu C, Qi X, Chen Y, et al. PI3K/Akt and MAPK/ERK1/2 signaling pathways are involved in IGF-1induced VEGF-C upregulation in breast cancer. J Cancer Res Clin Oncol. 2011;137(11):1587-1594. doi:10.1007/s00432-011-1049-2

42. Duffy MJ, Synnott NC, McGowan PM, et al. p53 as a target for the treatment of cancer. Cancer Treat Rev. 2014;40(10):1153-1160. doi:10.1016/j.ctrv.2014.10.004

43. Chao-Lin, Z. , Wei-Wei, X. , Yu, P. , et al. Same treatment for different diseases and individualized treatment for tumor based on gene. J Liaoning Univ of Trad Chin Med.(2012).

44. Shi, H.,W. Fu, Anti-gene Terapy and TCM Syndrome Diferentiation. World J IntegrTrad West Med 5, 563 (2004). 2004. 13(5): 563.

45. Changquan, L, W. Lina , W. Yuan, "The roles of traditional Chinese medicine in gene therapy. Chin J Integr Med. 12.2(2014):67-75.

\section{Figures}


A

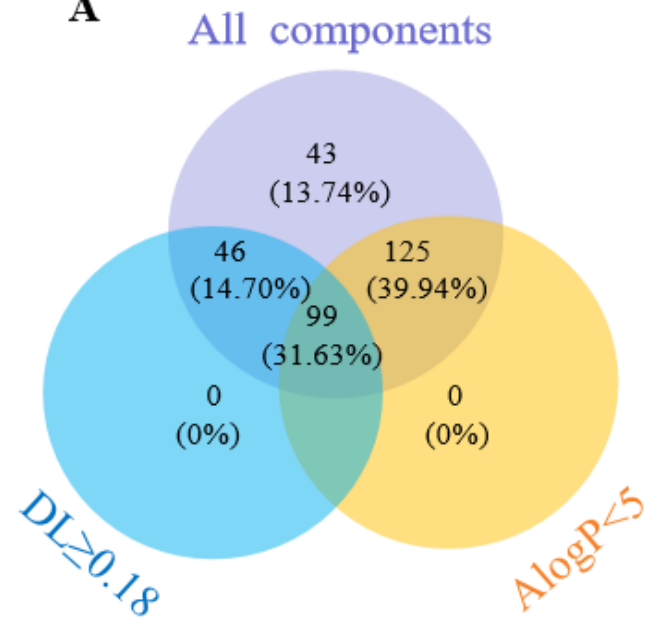

B

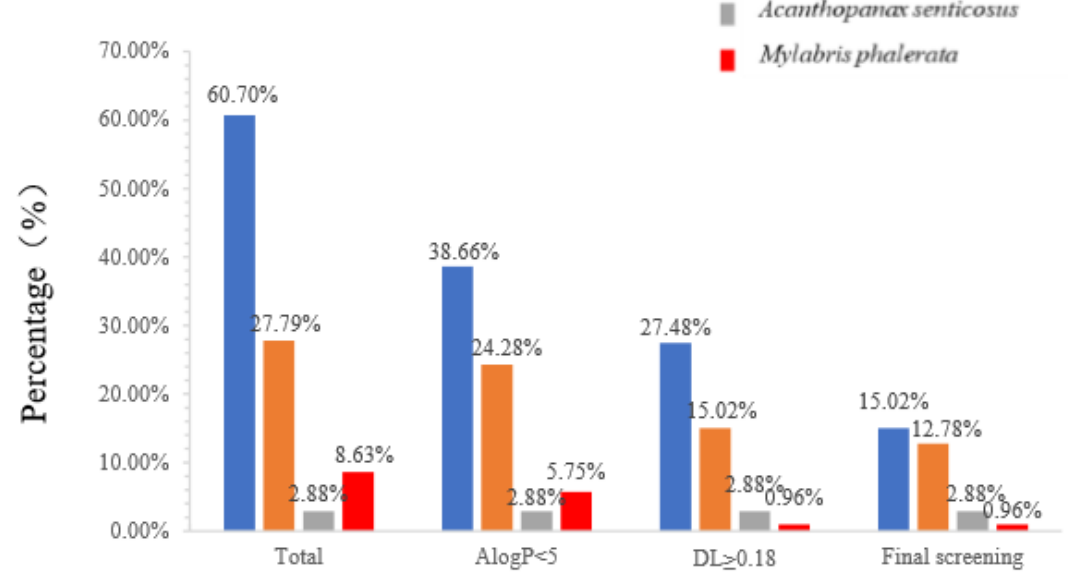

\section{Bioactive components screening}

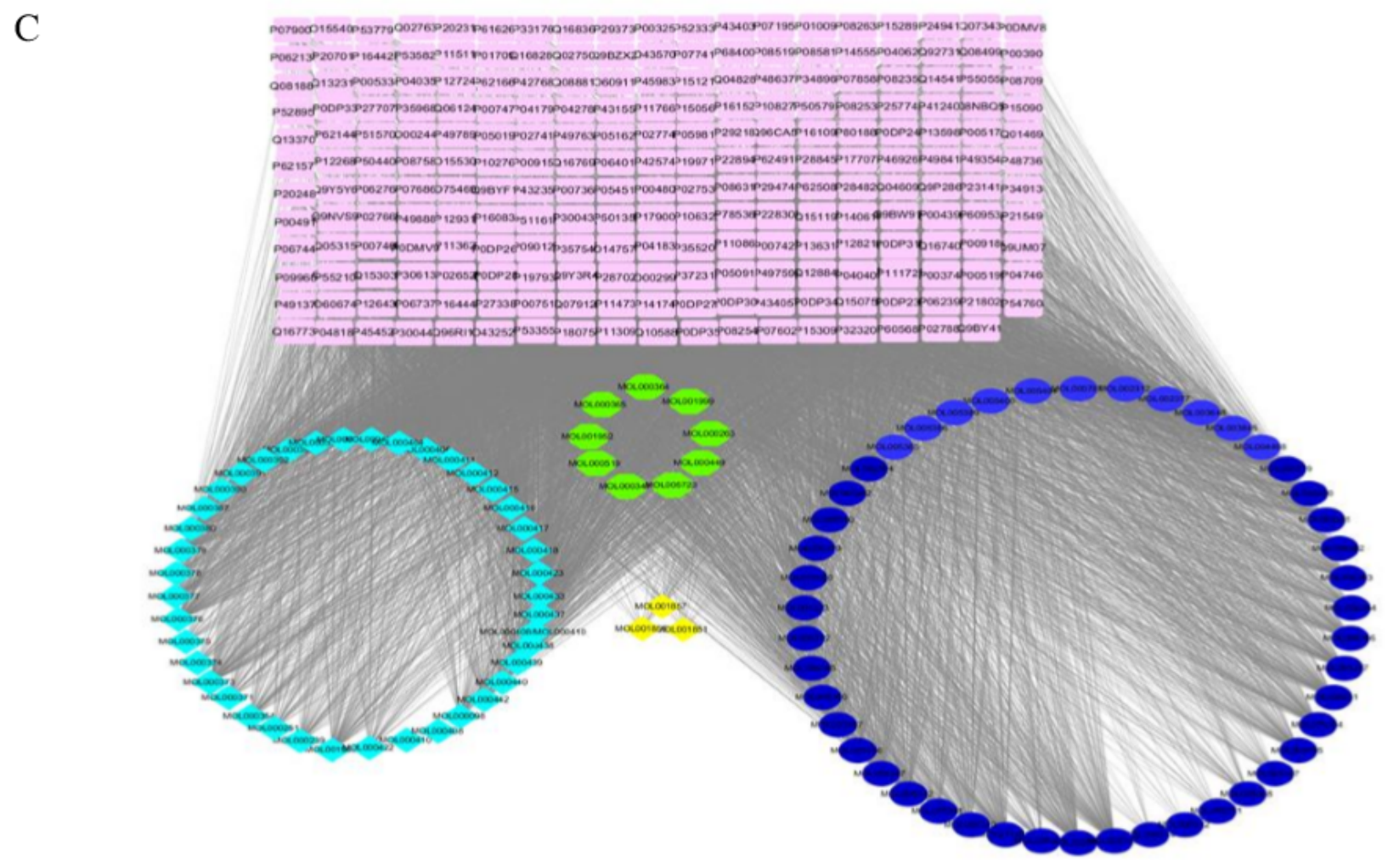

\section{Figure 1}

ADI component-target network. (A) Venn diagram: two ADME related models screen 99 bioactive components (blue section indicates AlogP $<5$ components, yellow section represents $D L \geq 0.18$ ).

(B)Distributions of different herbs. (C)Establishing a visual network of ADI components including 443 nodes and 863 edges. The blue, yellow, green and orange nodes represent bioactive components from renshen, astragalus, ciwujia and banmao, respectively. The pink nodes represent the target. 

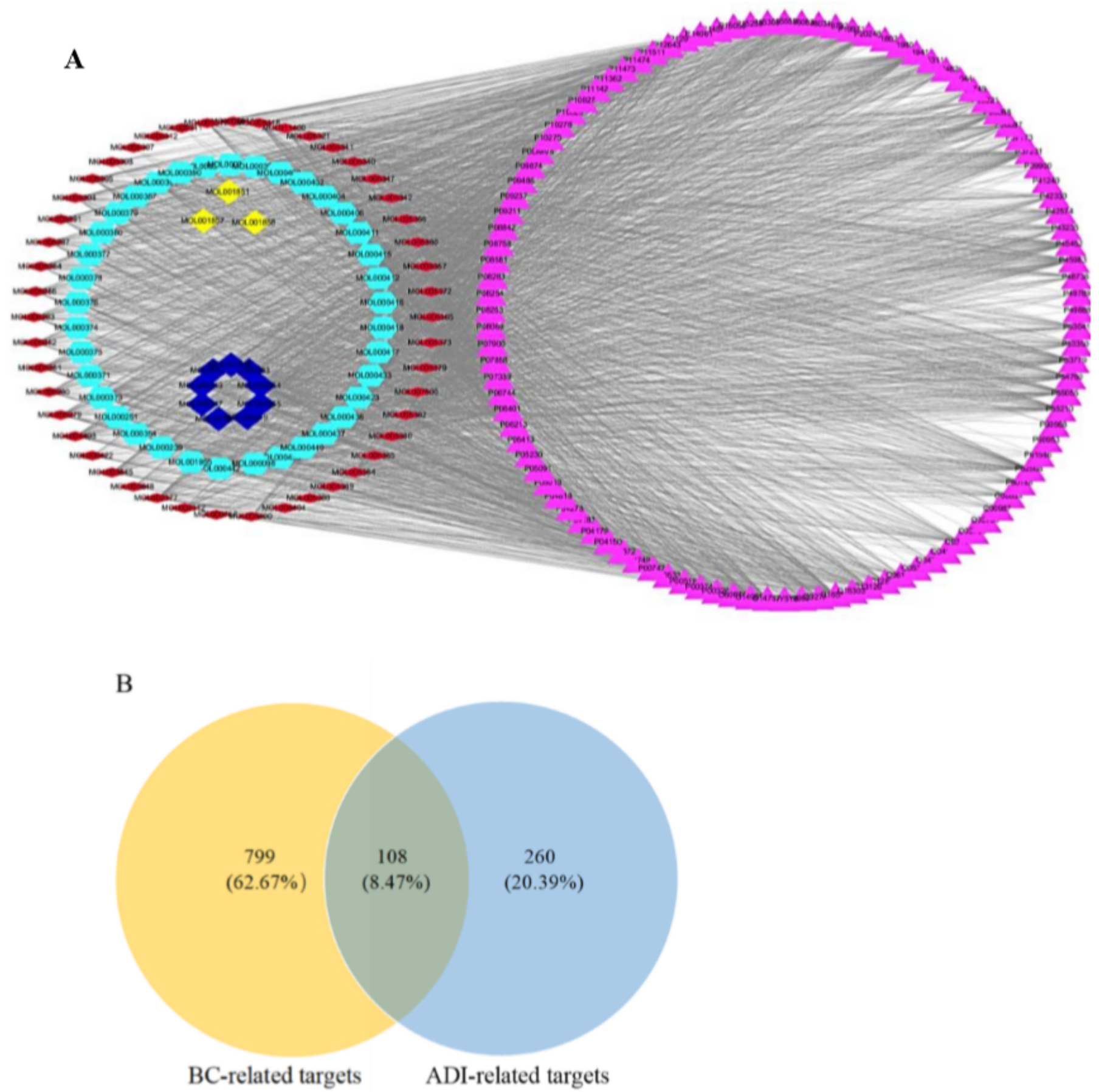

\section{Figure 2}

Common-target network. (A) The commom target network consists of 204 nodes and 3213 edges. The red, sky blue, blue and yellow nodes repre sent the bioactive components of Renshen, Huangqi, Ciwujia and Banmao, respectively, and the pink nodes represent the target. (B) ADI and BC have 108 common targets. 


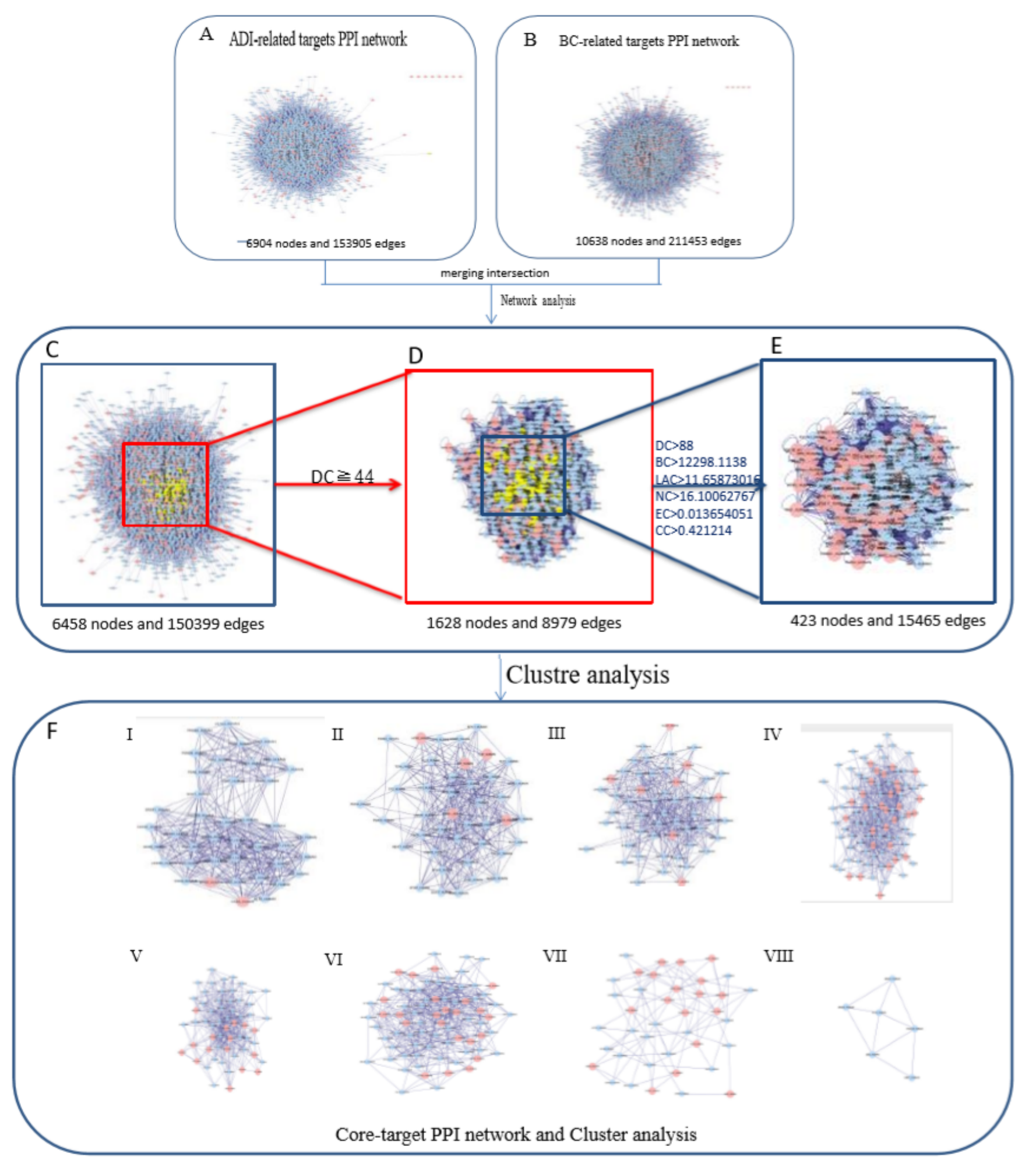

\section{Figure 3}

ADI-BC PPI network. (A) Targets PPI network related to ADI (6,904 nodes and 153,905edges). (B) Targets PPI network related to BC (10,638 nodes and 211,453 edges). (C) PPI networks intersection(6,458 nodes and 150,399 edges). (D) PPI networks using DC >44" as screening criteria (1,628 nodes and 18,966 edges). (E) Network with "DC">88区"EC">0.013654051囚"LAC">1165873016区"BC">11298.1138囚"CC">0.421214 and" 
NC">1610062767 as the core goal PPI screening criteria (423 nodes and 15,465,465 edges) .(F) Clusters of core-target PPI network. Pink indicates ADI related target and BC related target. yellow and blue indicate the selected target of screening criteria and other human proteins, respectively.

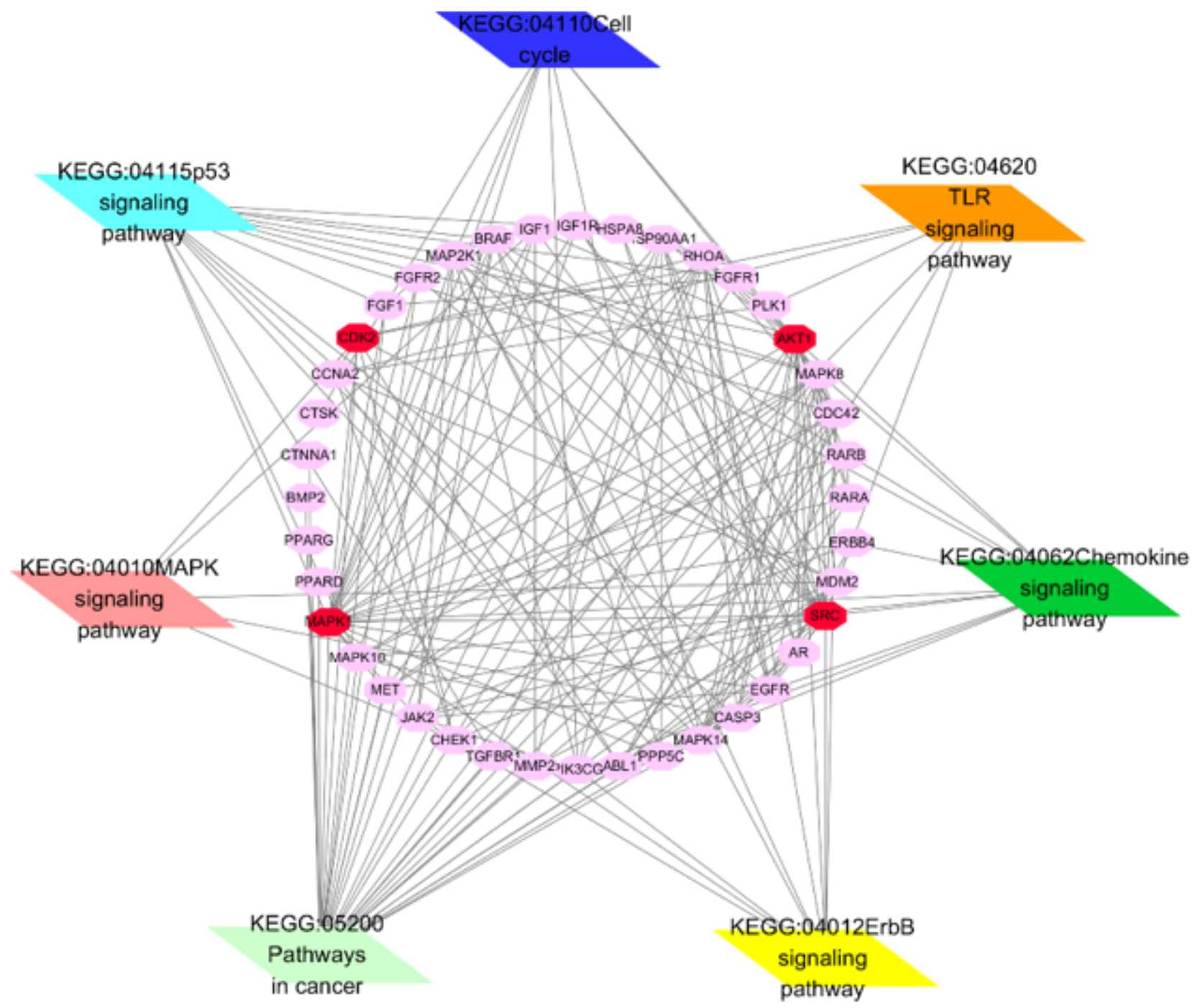

\section{Figure 4}

Core target path network. Sky blue, dark blue, purple, light green, yellow, dark green and orange nodes represent the seven signal paths of the main targets in the enrichment analysis; pink nodes represent the important common goals of $\mathrm{ADI}$ and $\mathrm{BC}$; red nodes represent the core common goals screened by the PPI network. 


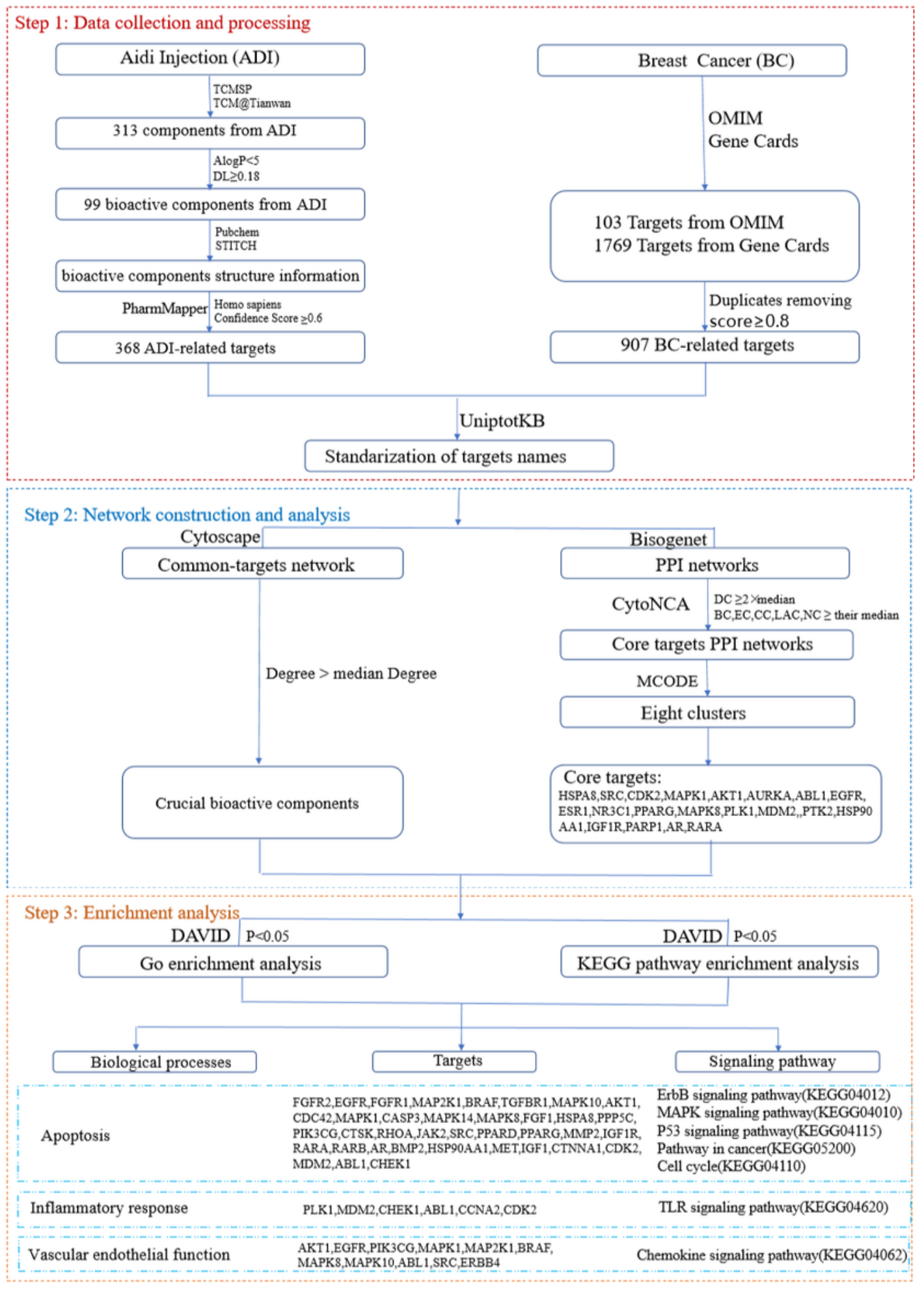

\section{Figure 5}

Diagram of the study design.

\section{Supplementary Files}

This is a list of supplementary files associated with this preprint. Click to download. 
- S1.ComponentsofeachherbinADI.xIsx

- S2.BioactivecomponentsofeachherbinADI.xIsx

- S3.PotentialtargetsofbioactivecomponentsinADI.xlsx

- S4.KnownBCrelatedtargets.xlsx

- S5.108commontargetsbetweenADlandBC.xIsx

- S6.Networkcentralityanalysisandevaluation.xlsx

- S7.GOandKEGGpathwayanalysisforthe108commontargets.xIsx

- S8.TheClusterofthecoretargetPPInetwork.xIsx

- S9.GOanalysisforeachcluster.xlsx

- S10.KEGGpathwayanalysisforeachcluster.xIsx 